\title{
CICRO: An Interactive Visual Interface for Crowd Communication Online
}

\author{
Masao Ohira, Hitoshi Masaki, and Ken-ichi Matsumoto \\ Graduate School of Information Science, Nara Institute of Science and Technology, \\ 8916-5, Takayama, Ikoma, Nara, Japan \\ \{masao, hitoshi-m, matumto\} @is.naist.jp
}

\begin{abstract}
As a means for online communication has become sophisticated and diverse (e.g. twitter, SNSs, YouTube, and etc.), a large indefinite number of users actively communicate each other in online communities. Thorough such the communications, they sometimes form the "crowd mind" which is temporally shared values and beliefs among mass users and can affect our society either positively or negatively. In this paper we present a concept of the online crowd and introduce CICRO, an interactive visual interface for the crowd communication. As a result of our experiment, we found that CICRO encouraged 1.7 times more active discussions among users than that of BBS since it provides users with an easy way to understand the existence of a variety of others' opinions and/or impressions in the discussions.
\end{abstract}

Keywords: Crowd communication, Visual Interface, Online community.

\section{Introduction}

As a means for online communication has become sophisticated and diverse (e.g. twitter, SNSs, YouTube, and etc.), a large indefinite number of users actively communicate each other in online communities. Thorough such the communications, they sometimes form the "crowd mind" which is temporally shared values beliefs among mass users and can work for our society either positively or negatively [1]. Although a small number of authorities (e.g., political party, government, religious organization and so on) and mass media have strongly influenced the formation of the crowd mind among people so far, people are currently able to be proactive in forming it by using internet and information technologies.

The crowd mind among people is not static but rather dynamic one. It can be changed over time depending on outside circumstances. A current interface for online communication, however, does not carefully consider the characteristics of such the user group and its communication. Most of online communication tools still provide users with traditional, text-based interfaces which cannot sufficiently capture or help the dynamical formation of the crowd mind. This situation might accelerate the "daily me" [2] phenomena where people prefer to get the information only they are interested in. It might not a democratic way of using the information technology [3].

Based on the findings from the literature of social psychology, in this paper we define such the online communication as "crowd communication" and try to show that 
the theory of the crowd mind in the physical (real) world can be applied to that formed online. We also introduce an interactive visual interface for the crowd communication, so called CICRO.

\section{Crowd Communication Online}

\subsection{The Notion "crowd"}

The notion "crowds" were invented through discussions among French social psychologists in order to explain the characteristics of large scale, collective behaviors of people, which had been observed saliently after the Industrial Revolution. Le Bon [1] described that the crowd is a group of people which is psychologically united by loosing sense of self from any cause. Tarde [4] used the notion "public" to describe the crowd clearer. The notion of public means a psychological collective formed among people who are physically distributed but intermediated by media such as news papers. In this sense, the crowd is a temporal group which is formed of massed people in a limited physical space and which acts together toward a certain shared goals. Summarizing these discussions, the crowd can be defined as follows:

- The crowds consists of a number of spatially-massed people,

- is a temporal group,

- shares a uniform environment, and

- is organized by common interests and situations defined by the environment.

The "crowd" in this study is also a temporal group of people which consists of massive online users on a virtual space. Different from the psychological unit (Tarde's "public") which is formed by the influences of one-way media such as news papers and TV, an online group shares common interests and situations on a uniform (virtual) environment by using interactive (bidirectional) online media such as chat and BBS. Their behaviors are constantly changing since the online group is affected by the summation of the interactions among the users. In this sense, the notion "crowd" in this study can be interpreted as the same notion of "crowd" in the social psychology, excepting the difference between a physical environment and virtual environment.

\subsection{Characteristics of Crowds}

In the literature of social psychology, the categorization of "crowd" and its formation mechanism has been studied [5, 6]. Table 1 shows the phenomenological classification of collective behaviors of crowds [5]. Roughly dividing crowds into two kinds of crowds (mob: active crowd and audience: positive crowd), the classification seems to succeed to create a consensus on understandings of crowds.

In contrast, we created the classification of online crowds as shown in Table 2. As Table 2 indicates, the classification of collective behaviors in a physical environment can be applicable to a virtual environment (online) since we can see that each type of the crowd exists even in a virtual environment. 
Table 1. Phenomenological classification of collective behaviors of crowds [5]

\begin{tabular}{|c|c|l|}
\hline \multicolumn{2}{|c|}{ type } & \multicolumn{1}{c|}{ example } \\
\hline \multirow{4}{*}{ mob } & offensive & crowd involving violent behaviors in disturbances \\
\cline { 2 - 4 } & escape & crowd escaping from disaster sites \\
\cline { 2 - 4 } & material & crowd rushing to panic purchases and deals to make a profit \\
\cline { 2 - 4 } & expressive & crowd enjoying to express feelings and impressions at festivals \\
\hline \multirow{2}{*}{ audience } & accidental & crowd consisting of third parties such as curious crowds \\
\cline { 2 - 4 } & intentional & crowd watching sports or listening to musical performance \\
\hline
\end{tabular}

Table 2. Collective behaviors of online crowds (massive user groups)

\begin{tabular}{|c|c|l|}
\hline \multicolumn{2}{|c|}{ type } & example \\
\hline \multirow{4}{*}{ mob } & offensive & user group causing framing on BBSs or blogs \\
\cline { 2 - 4 } & escape & user group worrying about the welfare of their family on BBSs \\
\cline { 2 - 4 } & material & user group rushing to online shopping sites to buy incorrectly-priced items \\
\cline { 2 - 4 } & expressive & user group posting their feelings and opinions to BBSs or blogs \\
\hline \multirow{2}{*}{ audience } & accidental & user group seeing accidental affairs such as flaming on BBS or blogs \\
\cline { 2 - 4 } & intentional & user group cheering for sports teams on micro blogs while watching TV \\
\hline
\end{tabular}

\subsection{Communication Media for Online Crowds}

Through the comparison of physical and virtual crowds, we were motivated to build a new interface for the crowd communication online because a current interface for online communication does not carefully consider the characteristics of online crowds and most of the online communication tools still provide users with traditional, textbased interfaces which cannot sufficiently capture and help the dynamical formation of the crowd mind.

As a first step toward creating communication media for online crowds, in this study we develop a visual interface for the crowd communication to meet the following requirements.

- It is easy to grape summations of opinions and impressions among users.

- It is easy to understand the scale and structure of conflicts between crowds.

- It provides users with a means to chime in the discussions among users (i.e. a means to quickly replicate the discussions in the past) for users joining the communication later.

In this study, we do not intend to cover all the types of crowds as shown in Table 2, but we would like to support the communication for the "expressive" crowd.

\section{CICRO: A Visual Interface for the Crowd Communication}

This section describes a visual interface for the online crowd communication we call CICRO (Communication Interface for CRows Online). Figure 1 shows CICRO's interface that visualizes the crowd communication in real time so that users can easily understand the scale of the crowd communication and relationships between opinions of the crowd. CICRO also has a function to replay crowd communications in the past 


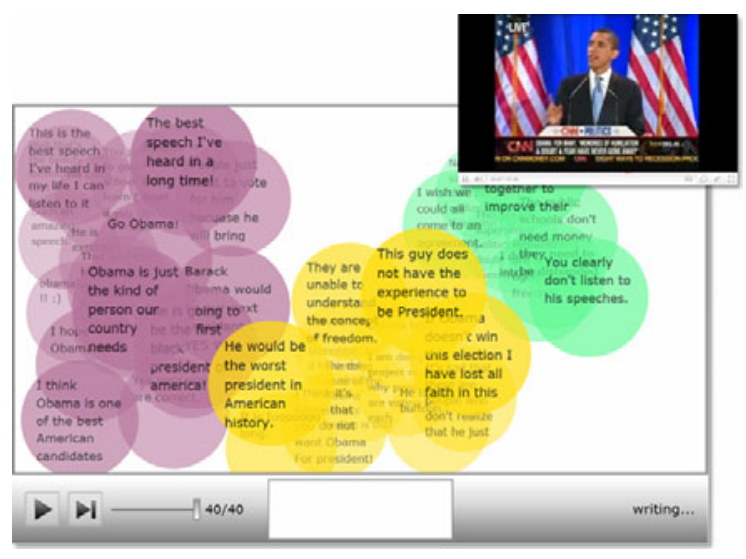

Fig. 1. A screenshot of CICRO. In this situation, users discuss about President Obama while watching a YouTube video. The groups of supporters and opponents are differently colored. Demos are available from http://crowdcom.sakura.ne.jp/hcii2011-demo/.

so that new users can quickly understand the past discussions among the crowd and join the current crowd communication. CICRO is built with Microsoft Silverlight 3 and runs on web browsers installed the Silverlight plug-in.

\subsection{Implementation}

Figure 2 shows the architecture and data flow of CICRO. CICRO consists of three components: CICRO Remark Controller for preprocessing input data (remarks) from users, CICRO Sever for storing the preprocessed data into a XML database, and CICRO Visualization Controller for interactively displaying the data to users.

Sending remarks. As shown in Figure 1, users send their remarks to CICRO by inputting the remarks with a keyboard and clicking any position to put them on the visualization area. CICRO Remark Controller takes remark data from users and coordinates data which is defined by the position users clicked. At the same time, it randomly determines colors of remarks on the visualization area according to the coordinates if users click a position where other remarks do not exist. It uses the color as same as existing remarks if users click on the existing remarks. After this, it sends the data to CICRO Server using HTTP.

Storing data. After CICRO Server receives the data from CICRO Remark Controller, it convert the data into the XML format using a custom Python script and then stores the XML data into a XML database.

Visualizing the crowd communication. CICRO Visualization Controller acquires remarks, coordinates, and colors data in real time from the XML database and stores it into the temporal data store which is always updated to have the most recent data up to 50 remarks. The canvas controller of CICRO Visualization Controller visualizes colored, circular remarks on the visualization area. 


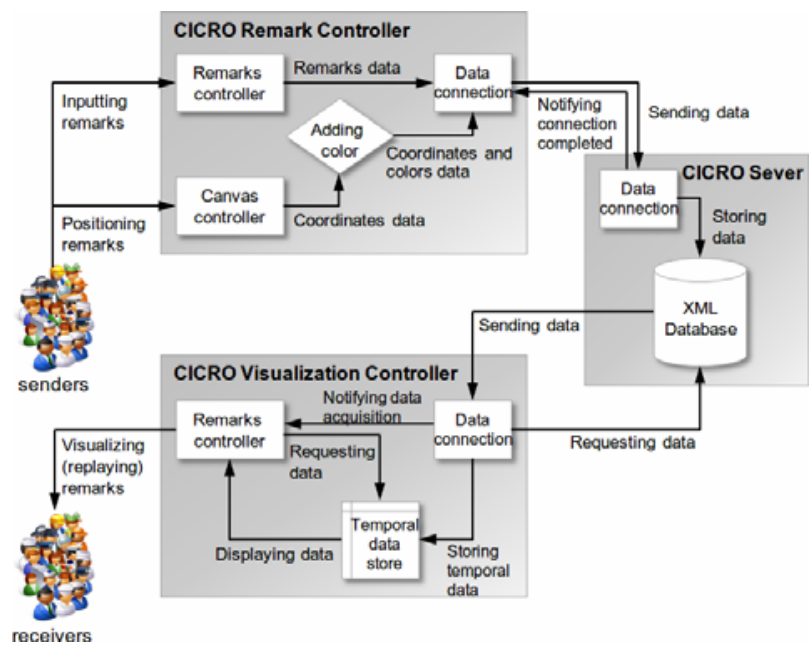

Fig. 2. Architecture and data flow of CICRO

\subsection{Interactions with CICRO}

Representation of a crowd. In the visualization of CICRO, "crowds" are represented as overlapped remarks with the same color, which mean that users put own remarks onto existing other users' remarks to express agreements and/or interests with other users' remarks (i.e., opinions and impressions). If a user disagrees with other users' remarks or other crowds, she can represent a counter opinion as a different colored remark by putting it on the blank space.

Anonymity. In the current implementation, CICRO represents a scale of a crowd not as the number of users but as the number of remarks. If a single user remarks again and again, one crowd can be formed only by the user. In contrast, if CICRO represents it as the number of users by coloring each user's remarks, each user will be identifiable from other users and it might result in hesitating to remark own opinions and impressions. This is a reason why we implement the way to visualize users' remarks anonymously. However, in the future we need to study this anonymity and its influence on the crowd communication.

Crowd communication in real time. In order to represent the crowd communication in real time, CICRO makes the size of an existing remark on the visualization area smaller little by little, each time a newer remark is created. In addition, CICRO only visualizes remarks up to 50 at the same time to avoid too complicated discussions among users. In the current implementation, 51th older remark is removed from the visualization area to display a new remark.

Replaying the crowd communication. For users who chime in the crowd communication, CICRO has a function to quickly watch the communication in the past. Users can randomly skip to any scene with the seek bar in the left bottom of the interface. 


\section{Comparative Experiment}

This section describes our comparative experiments to evaluate the usefulness of the CICRO's visual interface in the online crowd communication.

\subsection{Overview}

To evaluate the usefulness of the usefulness of the CICRO's visual interface in the online crowd communication, we conducted a comparative experiment where 34 subjects in total used CICRO and a common bulletin board system (BBS) respectively. While they watched a Japanese comedy show lively streamed on the internet, they used the two kinds of the communication interface to talk each other about the comedy show. After the session, we asked the subjects to answer a questioner on the usefulness of the two interfaces. We also counted the number of subjects' remarks in the session and analyzed (1) whether CICRO had an effect on the increase of the remarks or not, and (2) whether CICRO had an effect on the creation of the crowd mind among the subjects or not.

\subsection{Procedure}

Prior to the experiment, we recruited Japanese 34 undergraduate and graduate students (20-24 years old) as subjects from four different universities in Japan. We strongly prohibited them from telling anyone our experiment in order to ensure the anonymity of the subject during the experiment. We then divided them into two groups of randomly-selected 17 subjects. The subjects were asked to use his own computer to participate in the experiment where he could ensure the space without anyone else. The subjects were also asked to watch a Japanese comedy show lively streamed on the internet and to use both CICRO and BBS to freely discuss the show with other subjects who did not know each other.

Figure 3 shows the procedure of the experiments. The groups of the subjects participated in two sessions: one session for CICRO and the other session for BBS. In consideration of the order effect, the order of the interface use was counterchanged. Each session spent 45 minutes in total. In order to deeply look at the effects of the interfaces on online communication, we controlled that only the half subjects ( 8 or 9 ) of each group could join a session for first 10 minutes in the session. In this setting, we were interested in understanding which interface eased to chime in the discussions.

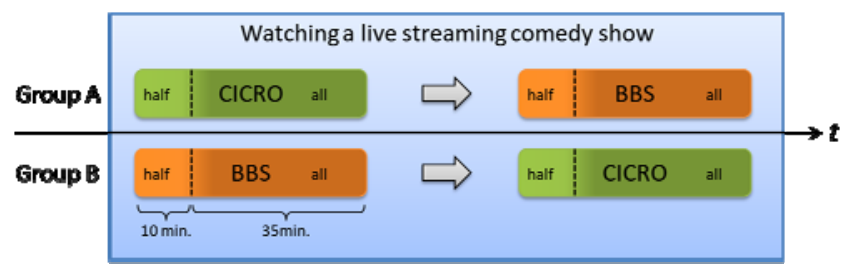

Fig. 3. Sessions in the experiment 
During the sessions, we recorded all the subjects' remarks on the two interfaces and captured subjects' screens. After the sessions, we asked the subjects to answer questioners on the usefulness of the two interfaces. We also interviewed them based on their answers.

\subsection{Results}

Number of remarks. Figure 4 shows the number of each subject's remarks in the sessions. The grayed bar is the number of remarks in using CICRO, and the white bar is the number of remarks in using BBS. We can see that the most of the subjects' remarks in using CICRO was larger than that in using BBS. In fact, the number of subject's remarks with CICRO was 1.7 times larger than BBS as shown in Figure 5 and Table 3.
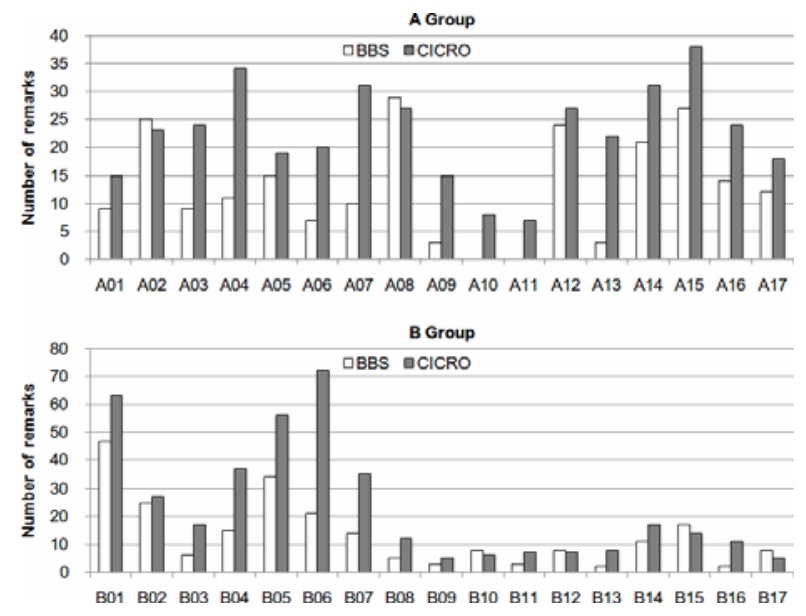

Fig. 4. Number of each subject's remarks in the sessions

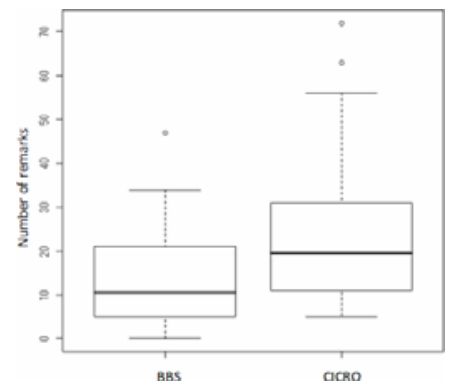

Fig. 5. Box plot of subjects' remarks in total 
Table 4 shows the result of the questionnaire\#1: "does the interface help you understand a variety of opinions and impressions in the sessions?" From this question, we tried to understand which interface is easier for the subjects to grasp others' opinions and impressions during the crowd communication. The result indicates that CICRO is superior to BBS for that purpose. The Wilcoxon signed-rank test also shows there was a significant difference between BBS and CICRO. Some of the subjects also answered our interview as follows:

- "I think one of the advantages of CICRO is that some topics can be discussed at the same time."

- "For colored remarks, I could intuitively (visually) understand responses from others."

Table 5 shows the result of the questionnaire\#2: "does the interface help you understand the scale of opinions and impressions in the sessions?" From this question, we tried to understand which interface is easier for the subjects to understand the existence of crowds and its scale. The result indicates that CICRO is also superior to BBS for that purpose. The Wilcoxon signed-rank test also shows there was a significant difference between BBS and CICRO. Some of the subjects answered our interview as follows:

- "It was fun because I could see that users was interested in the same topic and sharing the interests among each other."

- "It was easy to understand the extent to which similar opinions existed."

\section{Discussions}

The results of the comparative experiment showed that the visual interface of CICRO is superior to a traditional, text-based interface for the online communication since CICRO could motivate users to post their remarks (i.e., 1.7 times larger than BBS) and our subjects basically had positive impressions on CICRO. We believe we can successfully design the interface as we initially intended to help the communication for the "expressive" crowds.

One of the reasons why CICRO was better than BBS is that colored remarks on the interface triggered to express counter opinions and impressions from other users, resulting in active discussions among users. Another reason is that in the current implementation of CICRO, the size of older remarks gets smaller each time a new remark comes in the visualization area. Due to this limitation, if there are multiple groups (crowds) of users as shown in Figure 1, each group seems to be oriented to make remarks to keep their position more dominant or protect it in their discussions. We could observe such the interactions among our subjects at many times in case of using CICRO.

Although we could have positive outcomes from the experiment, we consider the results of the experiment as preliminary ones. Over several thousands of internet users sometimes communicate to discuss one topic at the same time. The results of our experiments were based on the analysis of the communications among only 17 subjects. This is obviously the limitation of our study in this paper. We need to scale up the user study in the future. To do so, we also need to scale up the functionality of CICRO as it can process and visualize a number of remarks at the same time. 
We are also aware that CICRO needs to automatically collect, group, merge, and polarize users' opinions. The current implementation of CICORO requires users to position their remarks manually. In order to overcome this inconvenience, we would like to incorporate natural language processing functions into CICRO in the future. In addition, we would like to develop several kinds of clients for the crowd communication, by extending existing communication tools such as twitter and YouTube. Furthermore, we would like to develop interfaces for other kinds of crowds described in Section 2, though in this paper we only present an interface for the communication of the "expressive" crowds.

\section{Related Work}

There have been many studies on the visualizations of online communications and communities. For instance, a variety of systems have been proposed to visualize conversations in a newsgroup. Newsgroup Crowds [7] and Netscan [8] provides a means to visualize the distribution of topics and users activities in a newsgroup. PeopleGarden [9] is a visual interface which employs a garden metaphor to represent enthusiasm for topics in a newsgroup. Loom [10] also visualizes threads of newsgroup conversations in a circular pattern to grasp active topics at the time.

These systems and interfaces are mainly designed to help users understand the current situation in a newsgroup and/or to motivate new users to join newsgroup discussions. Therefore, the conversations are visualized using the data in the past. CICRO places value on real-time visualization of the crowd communication to help "temporal" users better understand the scale and structure of the crowd communication. In this sense, Chat Circle [11], a chat system using the two dimensional remark positioning technique, seems to provide similar interactions to users as CICRO does. However, in Chat Circle, individual users are identifiable. It visualizes remarks so that users can easily find partners to talk with. In contrast, CICRO visualized remakes anonymously to encourage free discussions among users.

\section{Conclusions and Future Work}

In this paper we presented the concept of the crowd and its communication online. We introduced CICRO, an interactive visual interface for the crowd communication and showed the result of the comparative evaluation. We found that CICRO encouraged 1.7 times more active discussions among users than that of BBS since it provides users with an easy way to understand the existence of a variety of others' opinions and/or impressions in the discussions.

CICRO is currently designed to simply visualize the existence of different groups of online users with crowd minds. In the near future, we will built functions of the natural language processing into CICRO, in order to automatically collect, group, merge, and polarize users' opinions. We also would like to develop several kinds of clients for the crowd communication, by extending existing communication tools such as twitter and YouTube.

Acknowledgements. We would like to thank all of the students for participating in our experiment as subjects. 


\section{References}

1. Le Bon, G.: Psychology of Crowds (1895)

2. Negroponte, N.: Being Digital, Knopf (1995)

3. Sunstein, C.: Republic.com, Princeton University Press (2001)

4. Tarde, G.: L'opinion et la foule (1901)

5. Brown, R., Lindzey, G. (eds.): Mass Phenomena, Handbook of Social Psychology, vol. 2, pp. 833-877. Addison-Wesley, Reading (1954)

6. Turner, R.H., Killian, L.M.: Collective Behavior. Prentice-Hall, Englewood Cliffs (1957)

7. Viegas, F.B., Smith, M.: Newsgroup Crowds and AuthorLines: Visualizing the Activity of Individuals in Conversational Cyberspaces. In: Proceedings of the 37th Annual Hawaii International Conference on System Sciences (HICSS 2004), p. 40109b (2004)

8. Smith, M.A., Fiore, A.T.: Visualization components for persistent conversations. In: Proceedings of the SIGCHI Conference on Human Factors in Computing Systems (CHI 2001), pp. 136-143 (2001)

9. Xiong, R., Donath, J.: PeopleGarden: creating data portraits for users. In: Proceedings of the 12th Annual ACM Symposium on User Interface Software and Technology (UIST 1999), pp. 37-44 (1999)

10. Boyd, D., Lee, H.Y., Ramage, D., Donath, J.: Developing Legible Visualizations for Online Social Spaces. In: Proceedings of the 35th Annual Hawaii International Conference on System Sciences (HICSS 2002), vol. 4, p. 115 (2002)

11. Donath, J., Karahalios, K., Viegas, F.: Visualizing Conversation. In: Proceedings of the 32nd Annual Hawaii International Conference on System Sciences (HICSS 1999), vol. 2, p. 2023 (1999) 